\title{
Continuous Wavelet Transform: ECG Recognition Based on Phase and Modulus Representations and Hidden Markov Models
}

Lotfi Senhadji, Laurent Thoraval, and Guy Carrault

\subsection{Introduction}

Biomedical signals are fundamental observations for analyzing the body function and for diagnosing a wide spectrum of diseases. Information provided by bioelectric signals are generally time-varying, nonstationary, sometimes transient, and usually corrupted by noise. Fourier transform has been the unique tool to face such situations, even if the discrepancy between theoretical considerations and signal properties has been emphasized for a long time. These issues can be now nicely addressed by time-scale and time-frequency analysis.

One of the major areas where new insights can be expected is the cardiovascular domain. For diagnosis purpose, the noninvasive electrocardiogram (ECG) is of great value in clinical practice. The ECG is composed of a set of waveforms resulting from atrial and ventricular depolarization and repolarization. The first step towards ECG analysis is the inspection of $\mathrm{P}$, 


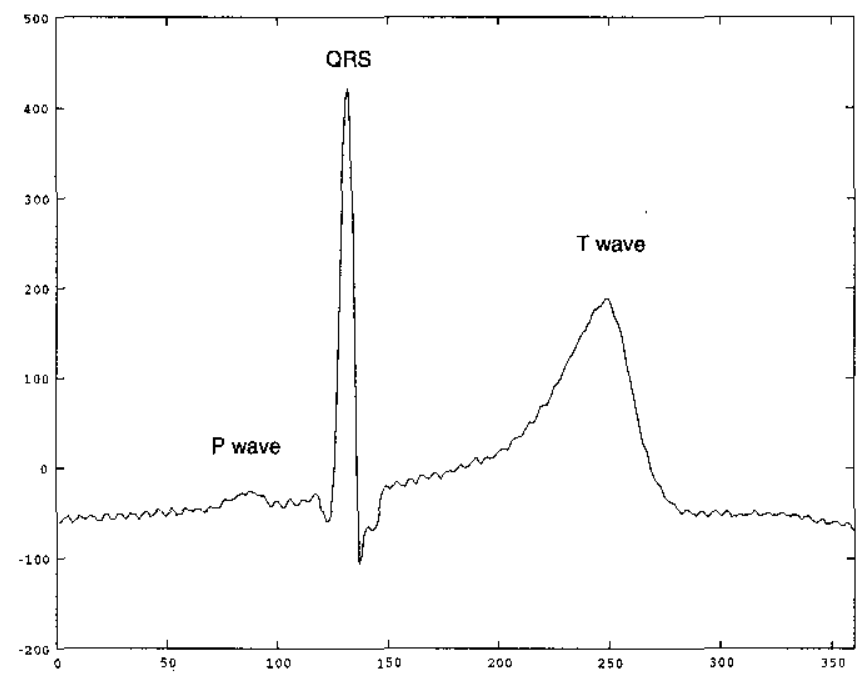

Figure 16.1

Example of a normal ECG beat.

QRS, and $\mathrm{T}$ waves; each one of these elementary components is a series of onset, offset, peak, valley, and inflection points (Figure 16.1). Ideally, the waves exhibit local symmetry properties with respect to a particular point (peak and inflection points locations of the considered wave). Based on these properties, one can extract significant points to study the wave shapes and heart rate variability [1].

Wavelet transforms have been applied to ECG signals for enhancing late potentials [2], reducing noise [3], QRS detection [4], normal and abnormal beat recognition [5]. The methods used in these studies were conducted through continuous wavelet transform [6], multiresolution analysis $[8,9]$ and dyadic wavelet transform [10]. In this chapter the continuous wavelet transform (CWT), based on a complex analyzing function, is applied to characterize local symmetry of signals, and it is used for ECG arrhythmia analysis. The first part of this chapter is more theoretical. The behavior of CWT square modulus of a regular signal $f(t)$ when the scale parameter goes to zero is studied. For a signal with local symmetry properties, the phase behavior of its CWT is also examined. These results are then extended, under some conditions, to signal without local symmetries. The second part is more experimental and numerical examples on simulated data illustrate the mathematical results. Finally, the use of these properties is considered in automatic ECG recognition and identification by means of hidden Markoy models (HMMs). The presentation emphasizes how a suitable parameter vector, corresponding to the input observation sequence of the Markov chain, can be built and applied. 


\subsection{Properties of Square Modulus and Phase}

With the same notations used in the first chapter of the book, the continuous wavelet transform (CWT) of a signal $f$ belonging to $L^{2}(\mathbb{R})$ is defined by :

$$
\left(W_{\Psi} f\right)(a, b)=\frac{1}{\sqrt{a}} \int_{-\infty}^{+\infty} f(t) \overline{\Psi\left(\frac{t-b}{a}\right)} d t
$$

where $\Psi$ is a complex valued function with zero mean and satisfying $C_{\Psi}<$ $\infty$ (see Chapter 1, Equation 1.24).

The wavelet of concern here is complex, compactly supported, hermitian (i.e., $\bar{\Psi}(t)=\Psi(-t))$, and $m$ times continuously differentiable $(m \geq 2)$. The analyzed signal $f(t)$ is real and supposed to be two times continuously differentiable.

\subsubsection{Square Modulus Approximation}

The square modulus of CWT of $f$ is defined by

$$
\left|\left(W_{\Psi} f\right)(a, b)\right|^{2}=\left(W_{\Psi} f\right)(a, b) \overline{\left(W_{\Psi} f\right)(a, b)}
$$

and its derivative according to the space variable $b$ is:

$$
\begin{array}{r}
\frac{\partial\left|\left(W_{\Psi} f\right)(a, b)\right|^{2}}{\partial b}=\frac{\partial\left(W_{\Psi} f\right)(a, b)}{\partial b} \overline{\left(W_{\Psi} f\right)(a, b)} \\
+\frac{\partial \overline{\left(W_{\Psi} f\right)(a, b)}}{\partial b}\left(W_{\Psi} f\right)(a, b)
\end{array}
$$

One can show that Equation 16.1 is equivalent to (see Appendix 1):

$$
\begin{array}{r}
\frac{\partial\left|\left(W_{\Psi} f\right)(a, b)\right|^{2}}{\partial b}=\frac{2}{a} \operatorname{Re}\left(\int_{-\infty}^{+\infty} f^{\prime}(t) \cdot \overline{\Psi\left(\frac{t-b}{a}\right)} d t\right. \\
\\
\left.\int_{-\infty}^{+\infty} f(t) \cdot \Psi\left(\frac{t-b}{a}\right) d t\right)
\end{array}
$$


where $\operatorname{Re}($.$) is the real part of its argument. At fine scales, the above$ derivative may be approximated by (see Appendix 2):

$$
\frac{\partial\left|\left(W_{\Psi} f\right)(a, b)\right|^{2}}{\partial b} \approx 2 a^{3} f^{\prime}(b) \cdot f^{\prime \prime}(b) \cdot\left|m_{1}\right|^{2}
$$

with

$$
m_{i}=\int_{-\infty}^{+\infty} x^{i} \cdot \Psi(x) d x \quad i \in \mathbb{N}
$$

According to this approximation, at fine-scale analysis, connections can be made between local extrema of $\left|\left(W_{\Psi} f\right)(a, b)\right|^{2}$ (as a function of the space variable) on one hand and inflection points and local extrema of $f$ on the other hand. Local maxima (minima) of $\left|\left(W_{\Psi} f\right)(a, b)\right|^{2}$ are always inflection points (local extrema) of $f$, but local minima may be also inflection points of $f$. These properties are summarized in Table 16.1 .

\subsubsection{Phase Behavior}

Based on the CWT modulus, one cannot, in general, recover the decomposed signal. The phase information is necessary to reconstruct the signal. In their pioneering work on the complex Hardy wavelet, Grossmann et al. [6-b] have indicated that phase information reveals isolated singularities (or local bursts) in a signal more accurately than does the modulus. In this section, the local behavior of the phase of CWT of signals with particular points as local extrema or inflection points is studied. We first consider the case of functions exhibiting local symmetry properties around these points, and then we extend the previous results to the case of an $m$ times continuously differentiable function $(m \geq 2)$.

Let $f(t)$ be a continuous function satisfying the following property:

$$
\left(\exists b_{0} \in \mathbb{R}\right)(\exists \varepsilon>0)(\forall|h|<\varepsilon) f\left(b_{0}+h\right)=f\left(b_{0}-h\right)
$$

which means that $f$ is locally symmetric with respect to the vertical axis crossing in $b_{0}$. (Note that if locally $f$ has no oscillations, $b_{0}$ is a local extremum.)

For fine scale, $\left(W_{\psi} f\right)\left(a, b_{0}\right)$ becomes (see Appendix 3, Case (1)):

$$
\left(W_{\Psi} f\right)\left(a, b_{0}\right)=2 \sqrt{a} \cdot \int_{0}^{+\infty} f\left(a t+b_{0}\right) \cdot \operatorname{Re}(\Psi(t)) d t .
$$

Hence, when the scale parameter " $a$ " goes to zero, $\left(W_{\Psi} f\right)\left(a, b_{0}\right)$ is real, and its phase is then 0 or $\pi$, according to the sign of (16.5). If $f$ has local 
Table 16.1

Summary of the properties of the CWT vs. local symmetry of the analyzed signal.

\begin{tabular}{l|l|l|}
\hline 1. Maximum $f(b)$ presents in $b_{0}$ a $\ldots$ & $\ldots$ then in $b_{0}\left|\left(W_{\Psi} f\right)(a, b)\right|^{2}$ is $\ldots$ \\
\hline 2. Minimum & Minimum \\
\hline 3. Inflection & Maximum \\
\hline 5. Inflection & Minimum \\
\hline
\end{tabular}

Note: For those regular shapes, local maxima of $\left|\left(W_{\Psi} f\right)(a, b)\right|^{2}$ point out the time locations of sharp transitions and correspond to two kinds of inflection points: (3) $f^{\prime \prime}\left(b_{0}^{-}\right)>0, f^{\prime \prime}\left(b_{0}\right)=0, f^{\prime \prime}\left(b_{0}^{+}\right)<0 ; f^{\prime}\left(b_{0}\right)>0$ (4) $f^{\prime \prime}\left(b_{0}^{-}\right)<0, f^{\prime \prime}\left(b_{0}\right)=0, f^{\prime \prime}\left(b_{0}^{+}\right)>0 ; f^{\prime}\left(b_{0}\right)>0$ 
symmetry property in $b_{0}$, then the phase of $\left(W_{\Psi} f\right)\left(a, b_{0}\right)$ is equal to 0 or $\pi$.

Suppose now that $f$, instead of obeying (16.4), has the following property:

$$
\left(\exists b_{0} \in \mathbb{R}\right)(\exists \varepsilon>0)(\forall|h|<\varepsilon) f\left(b_{0}+h\right)=2 f\left(b_{0}\right)-f\left(b_{0}-h\right)
$$

which indicates that $f$ is anti-symmetric around $f\left(b_{0}\right)$ (if $f$ is not locally oscillating, $b_{0}$ is a local extremum of $f^{\prime}$ ).

The assumption (16.6) implies the following equality at fine scales (see Appendix 3, Case (2)):

$$
\left(W_{\Psi} f\right)\left(a, b_{0}\right)=-2 i \sqrt{a} \int_{0}^{+\infty} f\left(a t+b_{0}\right) \operatorname{Im}(\Psi(t)) d t
$$

which means that the CWT of $f$ in $b_{0}$ is imaginary, with constant phase equal to $\pm \pi / 2$ according to the sign of (16.7). This property underlines that: if $f$ has a local center of symmetry in $b_{0}$ then the phase of $\left(W_{\Psi} f\right)\left(a, b_{0}\right)$ is equal to $\pi / 2$ or $-\pi / 2$.

The observed signal may not comply with symmetry assumptions but, even so, we still want to use the CWT tools to locate peak and inflection points. Let us now suppose that $f$ is an $m$ times continuously differentiable function $(m \geq 2)$; then, at fine resolution, $\left(W_{\Psi} f\right)\left(a, b_{0}\right)$ can be approximated by:

$$
\left(W_{\Psi} f\right)\left(a, b_{0}\right) \approx \sqrt{a} \cdot \sum_{n=1}^{m} \frac{a^{n}}{n !} \overline{m_{n}} f^{(n)}\left(b_{0}\right)
$$

where $f^{(n)}$ denotes the $n$th derivative of $f$. Using the Fourier Transform of $\Psi$ to express $m_{n},(16.8)$ becomes

$$
\left(W_{\Psi} f\right)\left(a, b_{0}\right) \approx \sqrt{a} \cdot \sum_{n=1}^{m}\left(\frac{a}{i}\right)^{n} \cdot \frac{\overline{\widehat{\Psi}}^{(n)}(0)}{n !} f^{(n)}\left(b_{0}\right)
$$

Hence, at high resolution (i.e., small value of " $a$ " or high frequency) CWT in $b_{0}$ is a linear combination of the analyzed signal derivatives. The variations of $\left(W_{\Psi} f\right)\left(a, b_{0}\right)$ across scales express the behavior of the derivatives of the signal according to the choice of the wavelet (consequently, all the moments $m_{n}$ are fixed). For example, if the first moment of $\Psi$ is null, asymptotically $f^{\prime}\left(b_{0}\right)$ does not influence the evolution of CWT in $b_{0}$ across scales. By limiting the approximation to the second order, we get:

$$
\left(W_{\Psi} f\right)\left(a, b_{0}\right) \approx-\sqrt{a^{3}} \cdot f^{\prime}\left(b_{0}\right) \operatorname{Im}\left(m_{1}\right) \cdot i-\frac{\sqrt{a^{5}}}{2} f^{\prime \prime}\left(b_{0}\right) \operatorname{Re}\left(m_{2}\right)=\alpha i+\beta
$$


where $\alpha$ and $\beta$ are real. It is then possible to associate particular values of the phase to local extrema and inflection points. For example, if a local extremum is reached in $b_{0}\left(f^{\prime}\left(b_{0}\right)=0\right)$, the phase is equal to 0 or $\pi$, depending on the sign of $\beta$ and, in the same way, a local inflection in $b_{0}$ $\left(f^{\prime \prime}\left(b_{0}\right)=0\right)$ is associated with phase value $-\pi / 2$ or $\pi / 2$, according to the sign of $\alpha$.

\subsection{Illustration on Signals}

In this section, numerical examples on synthetic data and real ECG signals are given to illustrate the above mathematical properties. The analyzing wavelet of concern is defined by (Figure 16.2):

$$
\Psi(t)=g(t) \cdot e^{2 i \pi k f_{0} t}
$$

where

$$
g(t)=\left\{\begin{aligned}
C \cdot\left(1+\cos 2 \pi f_{0} t\right) & \text { for }|t| \leq \frac{1}{2 f_{0}} \\
0 & \text { elsewhere }
\end{aligned}\right.
$$

The required admissibility conditions are satisfied for $k$ integer other than $-1,0,1$, and has been set to 2 . $f_{0}$ represents the normalized frequency $\left(0<f_{0}<1 / 2\right)$. For this wavelet, $\operatorname{Im}\left(m_{1}\right)$ is positive and $\operatorname{Re}\left(m_{2}\right)$ is negative.

\subsubsection{Results on Simulated Data}

In the following examples, $f_{0}=0.005$, the scale parameters are

$$
a_{i}=\frac{f_{0}}{f_{0}+i \cdot \Delta} \quad \text { with } \Delta=0.005,0 \leq i \leq 10 .
$$

\section{Example 1}

See Figure 16.3. The input signal (Figure 16.3a) behaves like

$$
A_{1} \operatorname{Exp}\left(-\frac{\left(t-m_{1}\right)^{2}}{b_{1}}\right)+A_{2}\left(t-m_{2}\right) \operatorname{Exp}\left(-\frac{\left(t-m_{2}\right)^{2}}{b_{2}}\right)
$$

with $A_{1}=15, b_{1}=1700, m_{1}=250 ; A_{2}=0.3, b_{2}=2500, m_{2}=625$. The associated CWT square modulus is reported (Figure 16.3b); the frequency 
increases (scale decreases) from the bottom to the top. We have reported on the " $Y$ " axis the parameter $i$ in place of the scale $a_{i}$.

The local extremal values of the square modulus make it possible to locate both the inflection points and the local extrema of the signal. This is clearly established Figure 16.1c, which shows the contour plot of the square modulus. Figure 16.1d depicts the phase variations between $\pm \pi$. As the phase is unstable when the modulus is close to zero, its value is fixed to zero when the modulus is less than a given threshold. Aligning the 0 and $\pi$ crossing of the phase from low to high frequency, one can localize the extrema of the signal, while $\pm \pi / 2$ are associated to inflection points.

\section{Example 2}

See Figure 16.4. Define $f_{0}(t)$ by:

$$
f_{0}(t)=\frac{1-\operatorname{Exp}\left(-\frac{\left(t-m_{1}\right)^{2}}{c}\right)}{2-\operatorname{Exp}\left(-\frac{\left(t-m_{2}\right)^{2}}{c}\right)}
$$

where $m_{1}=250, m_{2}=300, c=2500$. The signal used in this example is: $f(t)=A_{1} f_{0}(t)+A_{2} f_{0}^{\prime}\left(-\left(t+t_{0}\right)\right)$ with $A_{1}=10000, A_{2}=225$ and $t_{0}=10$. The symmetry properties do not hold in this case. However, inflection points and local extrema of the signal still can be localized using phase and modulus. According to the sign of $\beta$, the jump in the phase from $-\pi$ to $+\pi$ corresponds to a local maximum in the signal and zero crossing to a local minimum. As $\alpha>0$, inflection point on an increasing positive slope, corresponds to $-\pi / 2$ and on a decreasing negative slope to $+\pi / 2$.

\section{Example 3}

See Figure 16.5. The analyzed signal is the distribution

$$
U(t)= \begin{cases}1 & \text { if } t>t_{0} \\ 0 & \text { elsewhere }\end{cases}
$$

The associated CWT may be written as:

$$
\left(W_{\Psi} U\right)(a, b)=\sqrt{a} \int_{\frac{\left(t_{0}-b\right)}{a}}^{+\infty} \bar{\Psi}(v) d v
$$

Denote $\widetilde{\Psi}$, the function which is null except on the support of $\Psi$ and such that its derivative is equal to $\Psi$. Then the square modulus of Equation 
16.12 is equal to

$$
\left|\left(W_{\Psi} U\right)(a, b)\right|^{2}=a\left|\widetilde{\Psi}\left(\frac{t_{0}-b}{a}\right)\right|^{2}
$$

This quantity reaches its maximal value for $b=t_{0}$, and $\widetilde{\Psi}(0)$ is imaginary with phase equal to $-\pi / 2$ which means that CWT point out the time location of the jump in $U(t)$.

In practice, digital signal processing deals with input data obtained by analog low-pass filtering and a uniform sampling of a continuous time process. A signal like $U(t)$ is then smoothed (due to filtering) and becomes continuous at $t_{0}$ with a sharp transition (or maximal slope) at this point before the sampling procedure. WT of this sampled data behaves as for an inflection point on a positive increasing slope.

\subsubsection{Results on Real Data}

The signal of concern is a normal ECG sampled at $360 \mathrm{~Hz} ; f_{0}$ is set to 0.001 and the scale parameters are

$$
a_{i}=\frac{f_{0}}{f_{0}+i \cdot \Delta} \quad \text { with } \Delta=0.002,0 \leq i \leq 25 .
$$

\section{Example 4}

See Figure 16.6. From the CWT square modulus, the QRS and T waves are well localized because of their high slope, while $\mathrm{P}$ waves are not clearly separated from the QRS. In this example, the CWT has been multiplied by -1 before phase calculus in order to change the zero crossing into $\pi$ crossing. Because the phase is represented between $\pm \pi$, the $\pi$ crossing point corresponds to a discontinuity in the gray level representation (jump from black to white color). Using the phase representation, one can localize all the elementary components of the ECG when aligning the jump in the phase across scales. It can be seen that the phase locates the characteristic components of the signal more accurately; the modulus enhances the waves that have high slope (i.e., sharp waves), mainly the QRS and $\mathrm{T}$ waves, but does not allow the localization of $\mathrm{P}$ waves because of their low amplitude. To overcome this drawback, we proposed a nonlinear transformation (NLT) [11] to get transients with an inflection point corresponding locally to a potential wave peak and to enhance the energy of the $\mathrm{P}$ and $\mathrm{T}$ waves with respect to the QRS one (Figure 16.7). Local maxima of the signal is analyzed by means of CWT to exploit its inherent ability to point out the time location of such transients. 


\section{Example 5}

See Figures 16.8 and 16.9. The CWT is applied to the output of NLT. The wavelet transform has been multiplied by the complex $i$ to transform a $\pi / 2$ crossing in a color jump. $\mathrm{P}, \mathrm{QRS}$, and $\mathrm{T}$ waves can be clearly separated with the help of the CWT square modulus; the phase map led to the same results. Moreover, each curve of constant phase $\pi / 2$ varies according to the "propagation" across scales of the inflection point associated with a particular event in the signal. The energy distribution in the time-scale shows a similar behavior.

These remarks have been exploited from the pattern recognition point of view. The so-called "fingerprints" are here defined as the curves obtained by connecting, in the timescale domain, the points of a given constant phase value. In Example 4 (resp. 5), the fingerprints associated with the phase value zero (resp. $\pi / 2$ )-jump from black to white color-vary according to the shape of the corresponding events ( $\mathrm{P}, \mathrm{QRS}$, and $\mathrm{T}$ waves). Based on these remarks, a set of descriptors has been extracted from the timescale plane to characterize the elementary components of the ECG and to allow its recognition based on hidden Markov models.

\subsection{Cardiac Beat Recognition Approach Based on Wavelet Transform and HMMs}

Basically, hidden Markov models are doubly stochastic processes that can characterize any discrete sequence of feature vectors $\left\{\boldsymbol{o}_{t}\right\}_{1 \leq t \leq T}$, derived from an input signal $f(t)$ and considered as realizations of the so-called "observable process" $\left\{\boldsymbol{O}_{t}\right\}_{1 \leq t \leq T}$, by a set of multidimensional probability density functions (pdfs) whose parameters depend upon an unobservable first-order Markov state automaton or chain $\left\{X_{t}\right\}_{1 \leq t \leq T}$, the so called "hidden process". Practically, the hidden chain $\left\{X_{t}\right\}_{1 \leq t \leq T}$ models through its topology and its transition probabilities, the temporal and structural aspects of $f(t)$ while the state-dependent observation pdfs account for the probabilistic nature of the feature vectors from which they are derived. For both reasons, hidden Markov modeling is well suited to the analysis of structured random signals that are essentially segmental in nature. After designing several competing models of arrhythmias, beat classification and labeling, and/or complex rhythms, analysis can be performed based on dynamic programming techniques and the maximum likelihood criterion. The modeling techniques described here consider the observed ECG signal $f(t)$ as being equivalent to a sequence of events associated with state changes. 

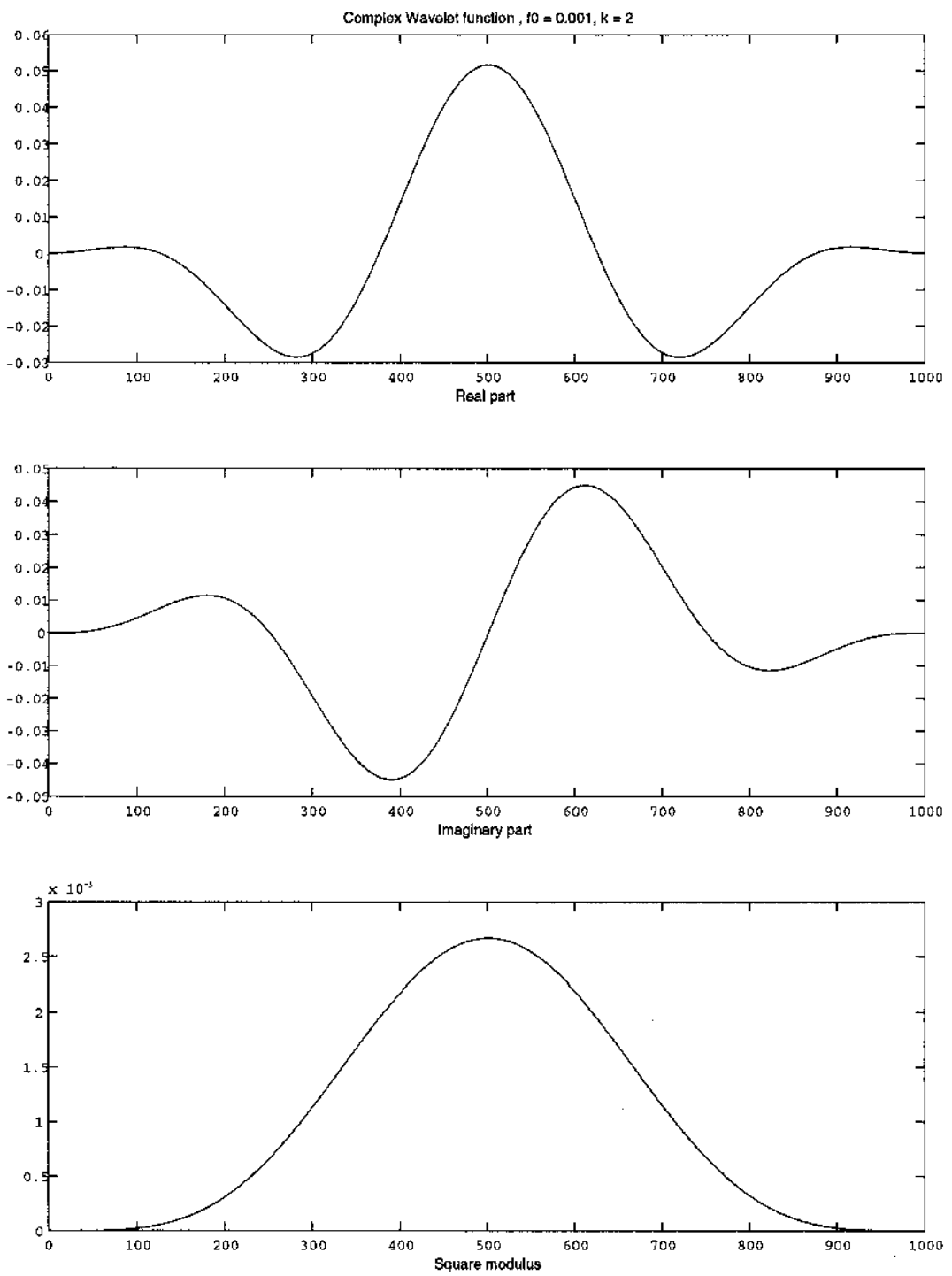

Figure 16.2

The plot of the real part, imaginary part, and square modulus of the wavelet used. 

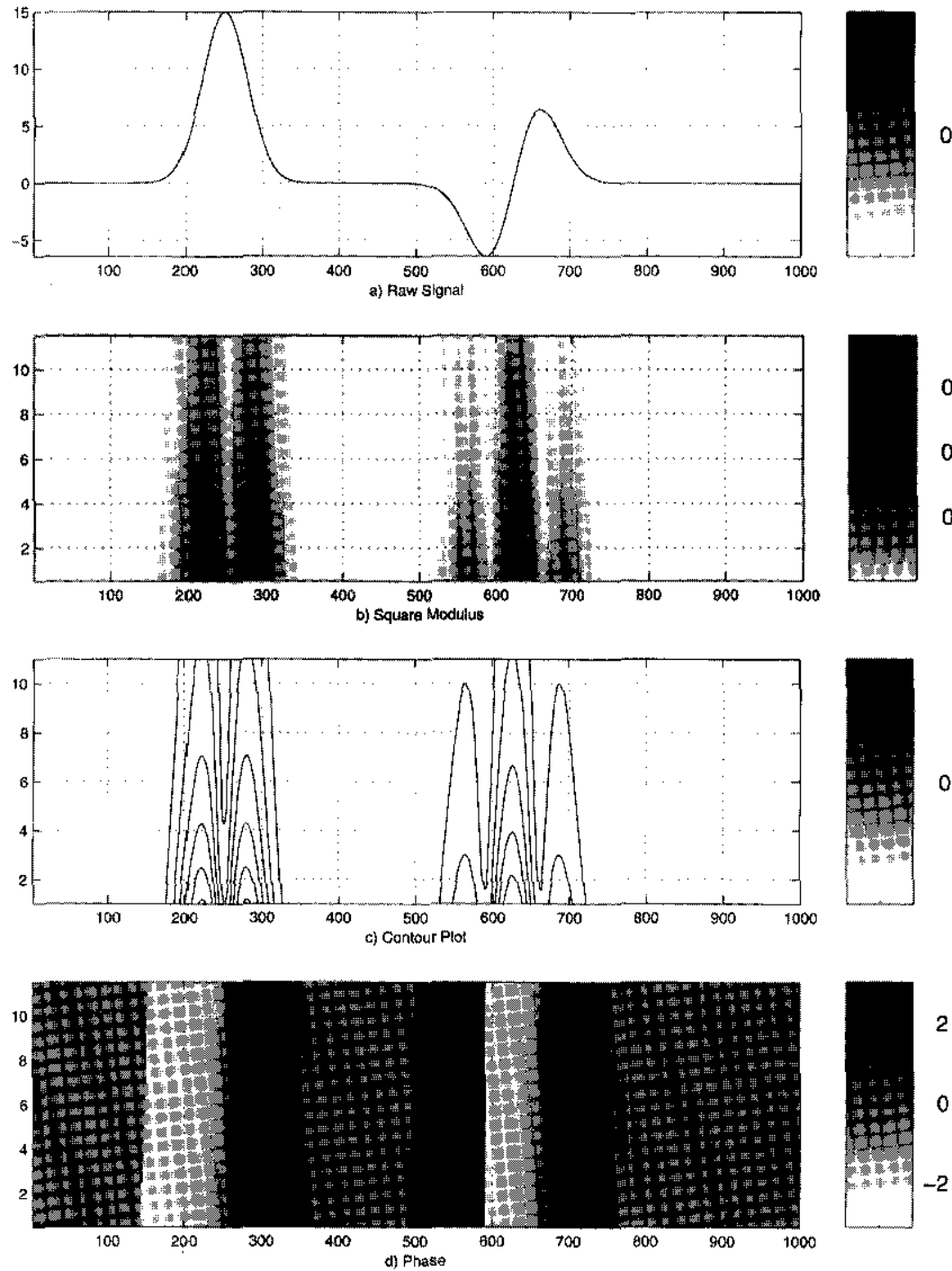

Figure 16.3

CW'T of simulated data with local symmetry properties. 

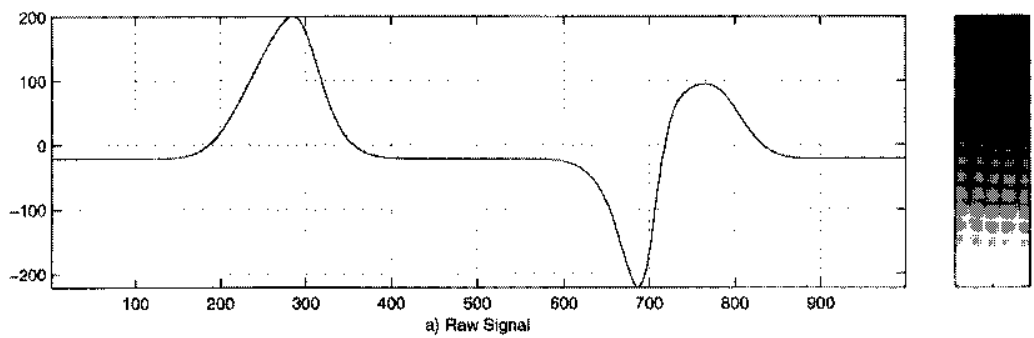

1

0.5
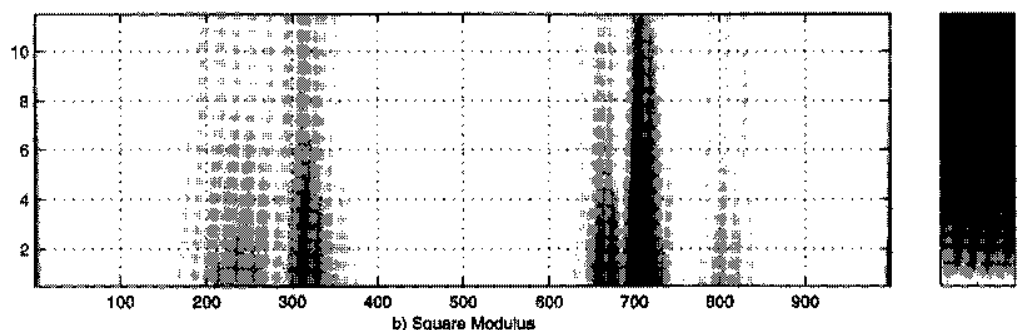

300

200
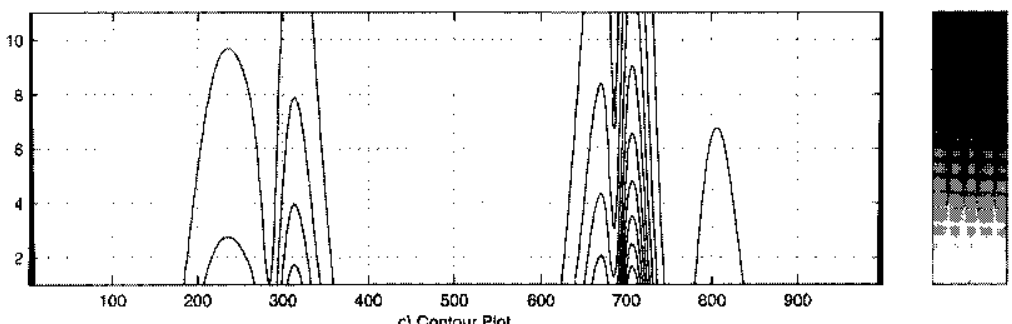

1

0.5
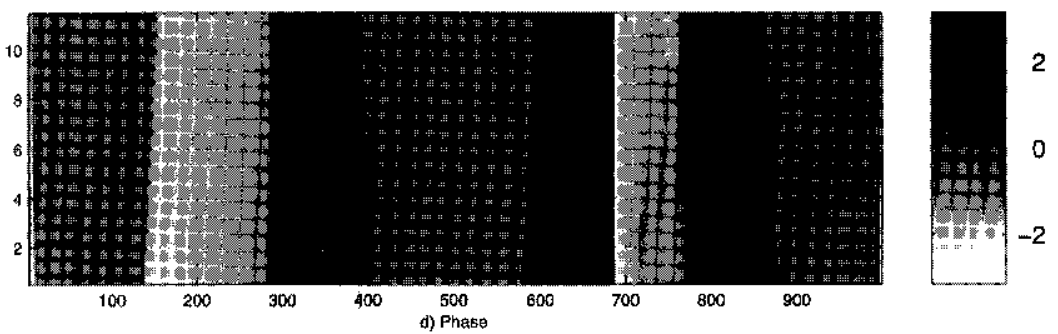

Figure 16.4

CWT of simulated data without symmetry properties. 

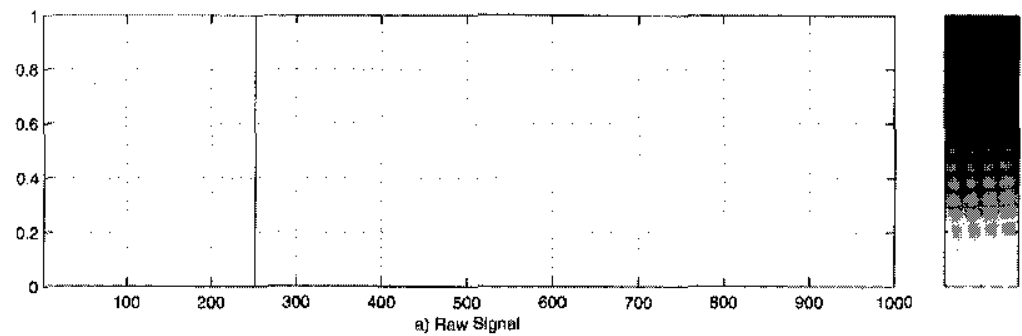

1

0.5

0
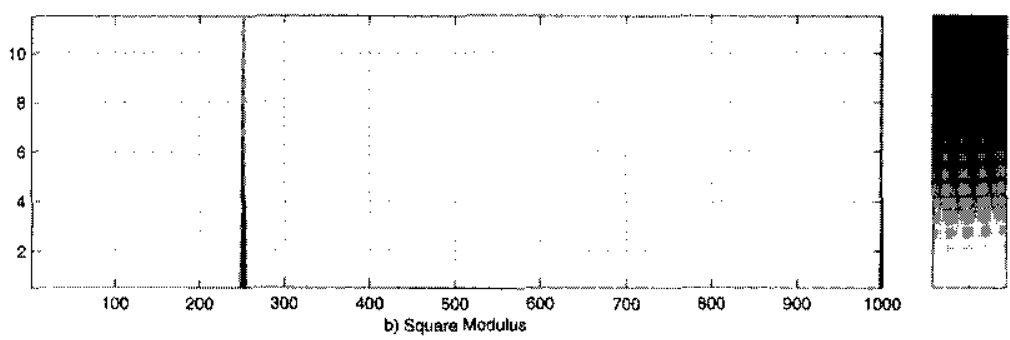

0.6

0.4

0.2
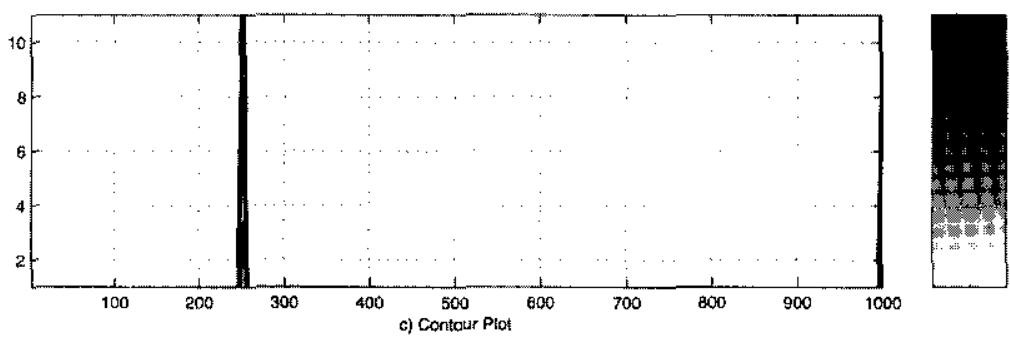

1

0.5

0
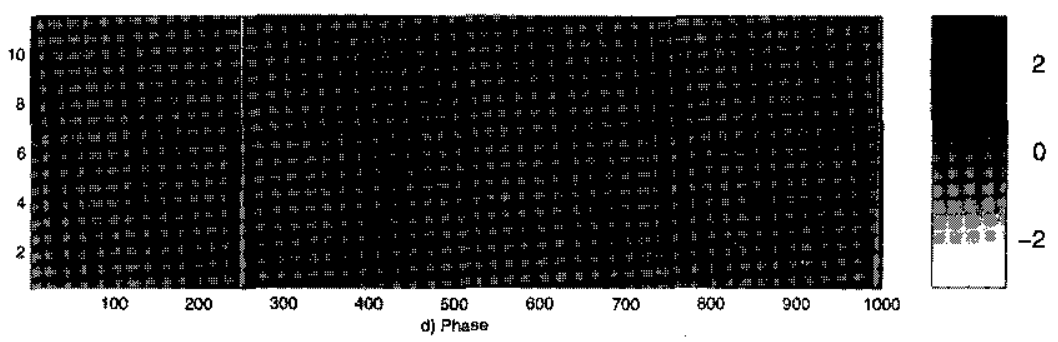

Figure 16.5

CWT of a simulated signal with a particular discontinuity. 

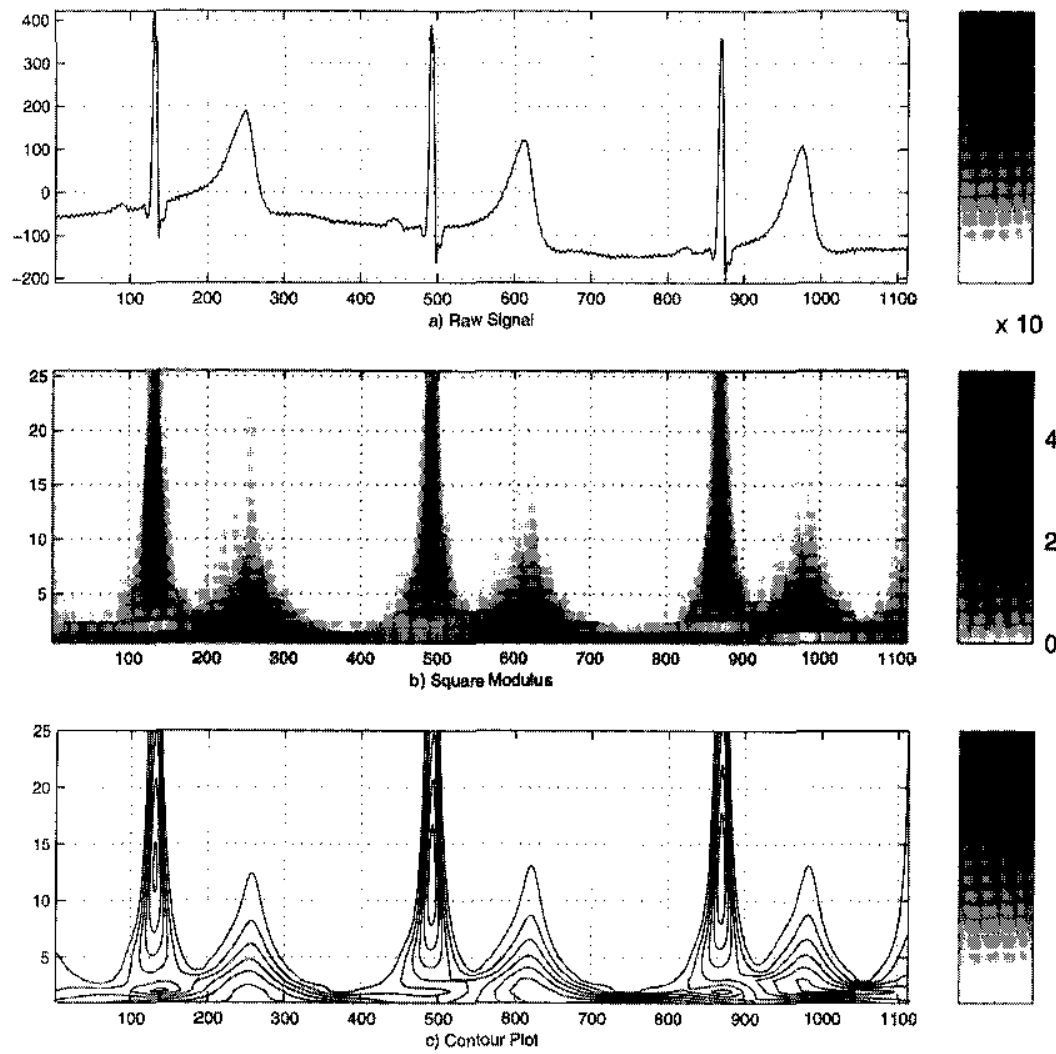

1
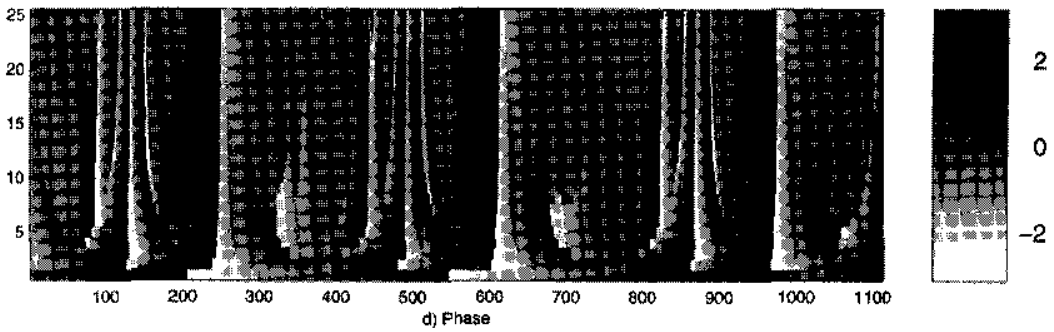

Figure 16.6

CWT of real ECG. 

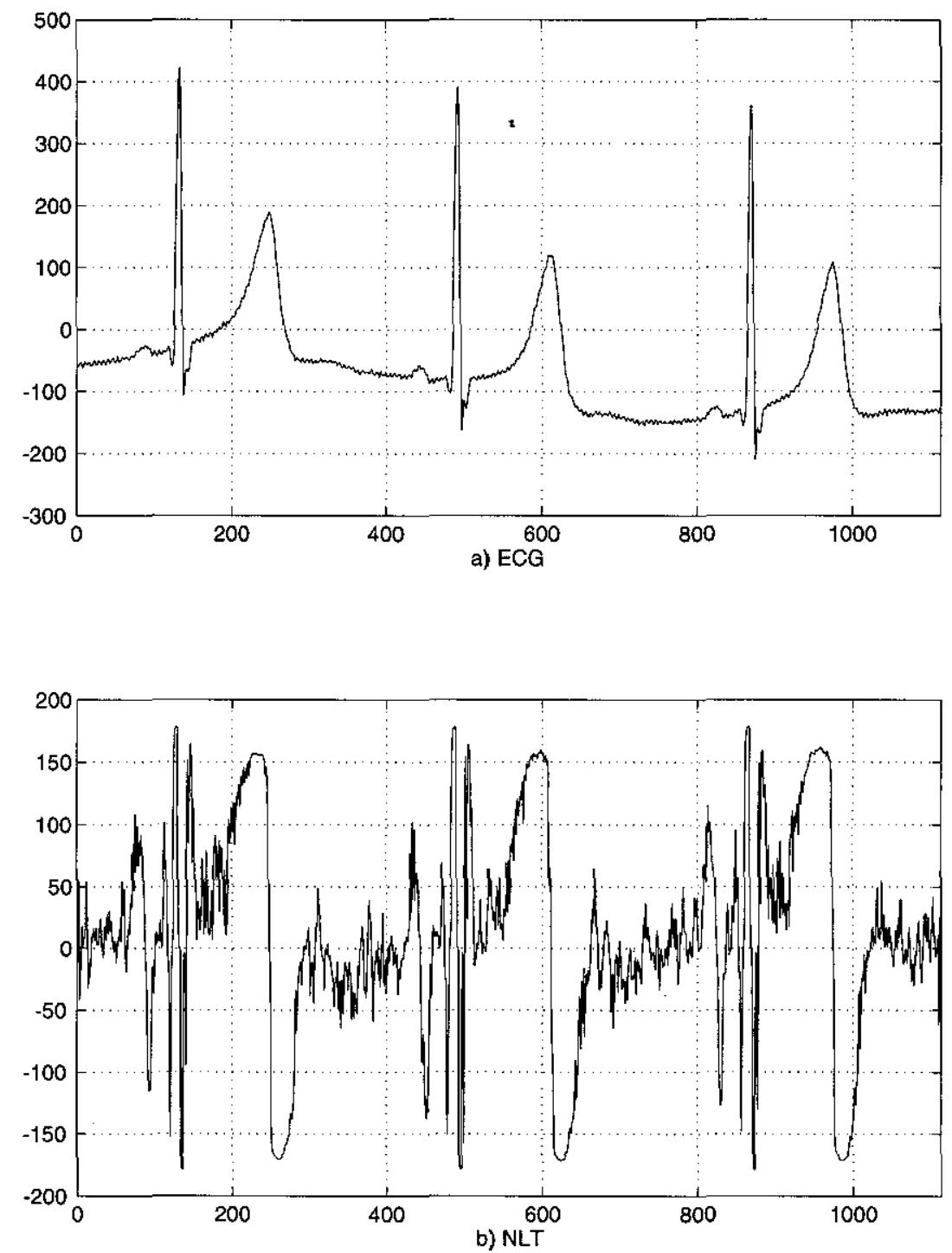

Figure 16.7

Example of the NLT procedure applied to the ECG. 

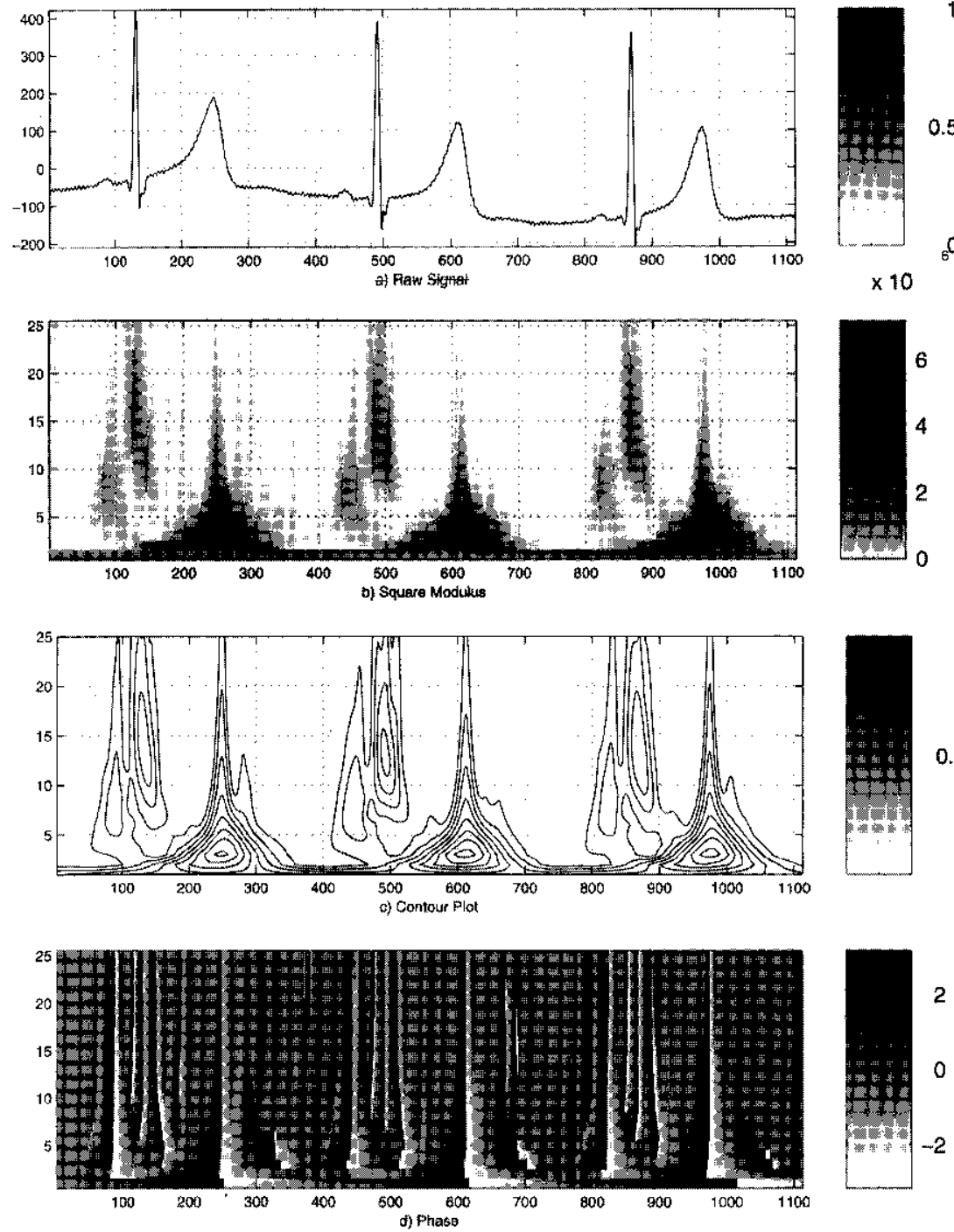

Figure 16.8

CWT performed on the NLT of the ECG. 

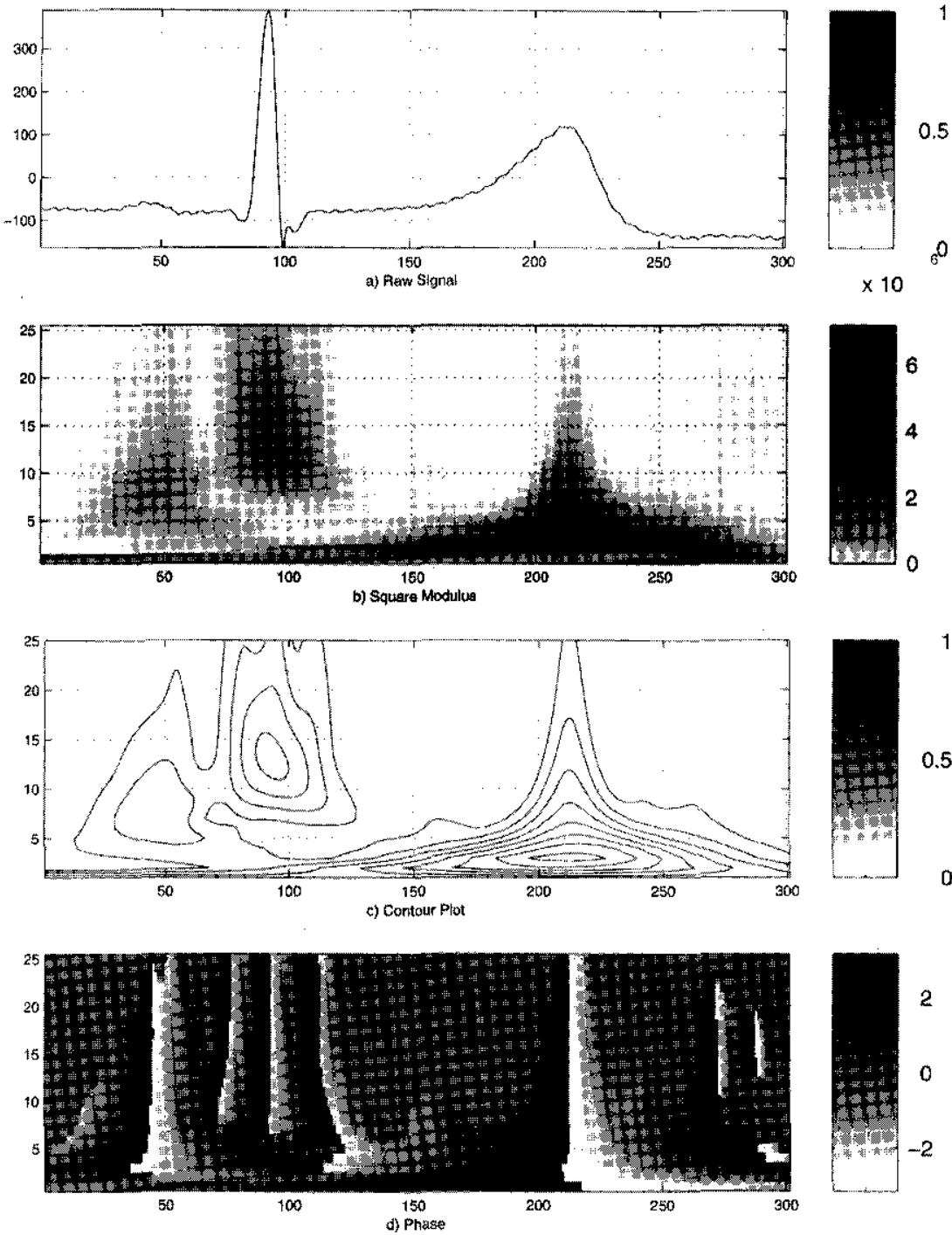

Figure 16.9

Close-up view of the second ECG beat of the Figure 16.8. 


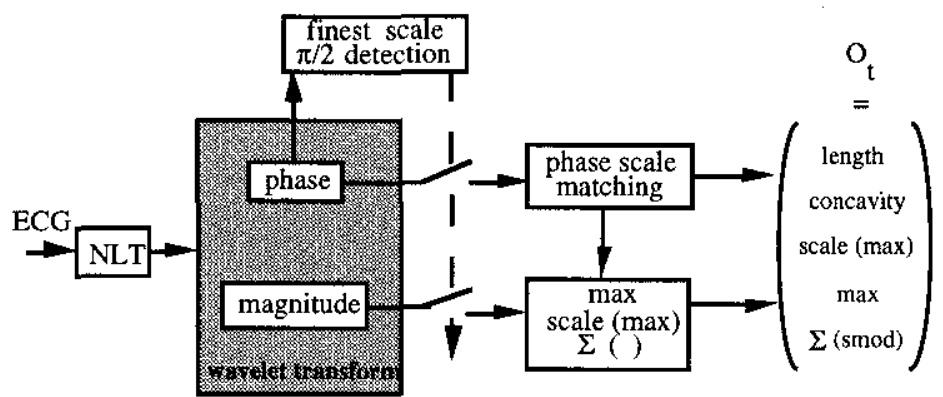

\section{Figure 16.10}

\section{Block diagram of the data preparation procedure.}

In practice, a preprocessing stage first projects the observed ECG signal $f(t)$ into a discrete sequence of feature vectors $\left\{\boldsymbol{o}_{t}\right\}_{1 \leq t \leq T}$. Then, the likelihood of $\left\{\boldsymbol{o}_{t}\right\}_{1 \leq t \leq T}$ is simultaneously assessed by several competing models: their respective states describe the different signal patterns that can occur in the rhythmic disorder associated with, and their topology depicts the statistical arrangement existing between them along the time axis. The objective here is to show how the wavelet transform, based on the suitable properties described previously, could be used in the ECG data preparation step (more precisely in the construction of the observation vector $\boldsymbol{o}_{t}$ ). Markov theory is not reported here; the reader may refer to [12].

The data preparation is depicted in Figure 16.10 and relies on the extraction of shape parameters from CWT performed on the output of the NLT of the ECG. As seen before, for each elementary wave, the NLT generates transients with an inflection point corresponding locally to its dominant extremum. The ability of the CWT to focus on the edges of a signal is here of great interest. The combination NLT/WT allows enhancement of the separation between the elementary waves in the scalogram (Figure 16.9). Moreover, each fingerprint characterizes, by its length, the persistency of an event across the scales and, by its concavity, its relative time position in the signal.

For each $\pi / 2$ crossing of the phase at the finest scale of decomposition and time position $t$, the associated fingerprint is reconstructed by an ad hoc procedure under a simple constraint of continuity; then an observation vector $\left\{\boldsymbol{o}_{t}\right\}$ is derived. It is composed of five parameters:

- "Length": the length of the fingerprint in terms of number of associated levels of decomposition;

- "Concavity": its concavity;

- "scale (max)": the scale value, over the support of the fingerprint, where the CWT square modulus is maximum; 


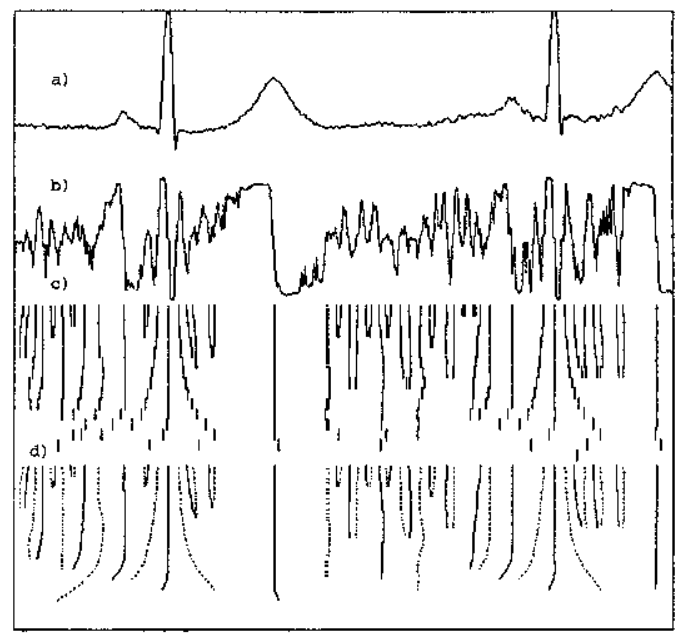

Figure 16.11

a) ECG signal, b) the corresponding NLT, c) fingerprints on the time-scale domain, d) the reconstructed fingerprints.

- the magnitude of the above maximum "max";

- the summation of all the CWT modulus on the fingerprint, " $\Sigma(\operatorname{smod}) "$.

\subsection{Results}

In our experiments, the phase is computed over a finite scale set in order to derive relevant fingerprints; at each $\pi / 2$ crossing, the NLT is then decomposed on several levels to recover correctly the theoretical bandwidth of elementary waves. The transition probabilities of the models are set equal to consider as equiprobable the presence or the absence of interwave observations. The five parameters composing the observation vectors $\boldsymbol{o}_{t}$ are assumed to be independent. Moreover, "length", "concavity," and "scale (max)" are discrete random variables while "max" and " $\Sigma(\mathrm{smod})$ " are Gaussian. The wave couplet durations are modeled by truncated Gaussians so that they are lower and upper bounded. All the parameters of the probability laws are initialized with a clustering of a small observations set and then refined cyclically using a modified version of the Baum-Welch's reestimation procedure [13].

Figure 16.11 depicts an ECG signal with its corresponding NLT, the fingerprints before and after reconstruction (represented on 15 levels). Thus, 

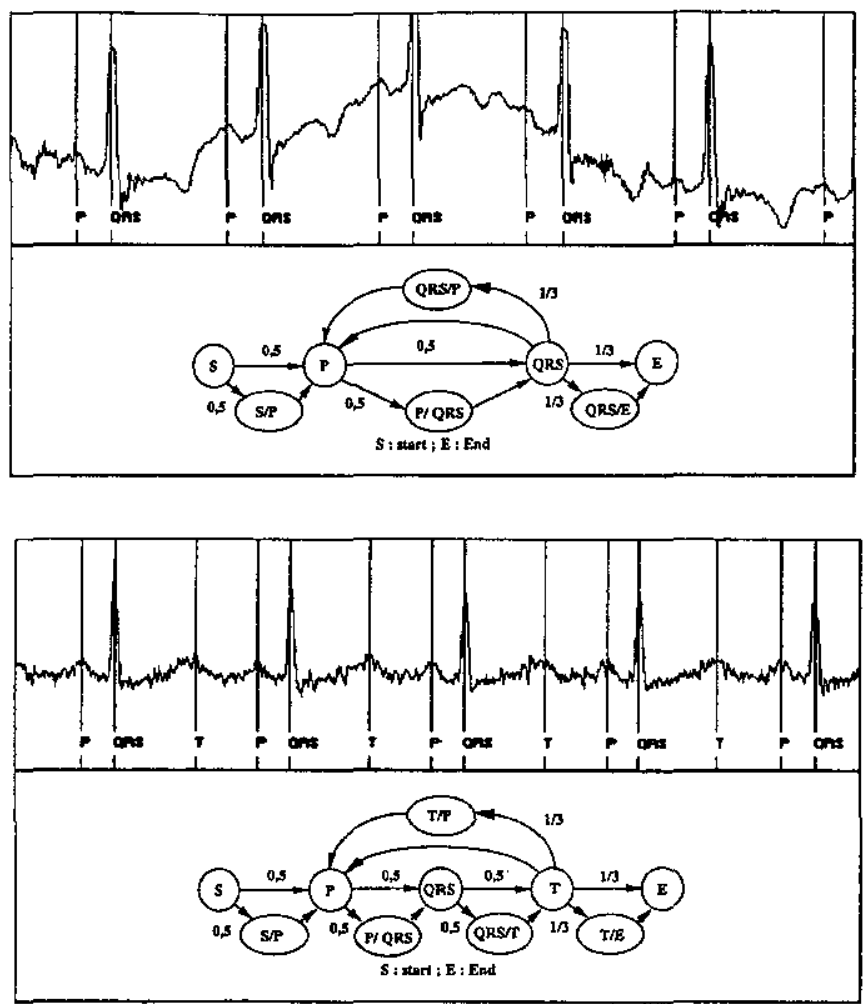

Figure 16.12

Two examples of the structure of the Markov state chain used to decode the corresponding observation sequence derived from signal by data preparation stage. Vertical lines locate the elementary waves recognized by the structure.

for each time candidate an observation vector is derived from $f(t)$. Finally, the resulting observation serie is processed by the hidden chain depicted in Figure 16.12. A transition between " $\mathrm{T}$ " and "P" states via the "T/P" one is allowed to process successive cardiac beats. "S" and "E" states model the onset and the end of the signal. Note that all ECG constitutive waves are well localized and identified even when HF noise, mainly due to the electromyography activity, is present. In our ECG recognition process, the strong nonstationarity of the signal is shaped essentially because each wave is viewed as a unique stationary entity, rather than a locally stationary stochastic process. Although our approach is still under test, the first results obtained show that the recognition rate is enhanced compared to the procedure where segmentation and identification of ECG waves are performed simultaneously by classical HMMs. 


\subsection{Conclusion}

We have presented some properties of a complex wavelet transform. The modulus maxima and the $\pm \pi / 2$ phase crossing point out the locations of sharp signal transitions, while modulus minima correspond to the "flat" segments of the signal. The results on simulated data show that phase information may be of great interest when time location of particular events of such peaks is looked for. On ECG signal, the behavior of both phase and modulus of the decomposition when the scale goes to zero allows description of the elementary components ( $\mathrm{P}, \mathrm{QRS}, \mathrm{T}$ ). By exploiting these properties, the Markov models, briefly presented here, can behave as a segmentation/recognition signal processing tool, achieving the numbering of all the waves (including the $\mathrm{P}$ ones), by probabilistically modeling the temporal structure of the observed surface ECG. The set of standard mathematical tools devoted to the use of HMMs constitutes a found theoretical basis. It must be emphasized that there is no restriction on the use of Markovian models when the physical phenomenon is only approximately Markovian. It must be said, however, that the segmentation of the observed signals may not be sufficient to identify some pathological situations, for instance, when the waves, say $\mathrm{P}$ and QRS, usually appearing on different time intervals, are superimposed, as in the auricular-ventricular dissociation.

\subsection{Appendix}

\section{Appendix 1: Expression of CWT Square Modulus Derivative}

The derivative of $\left(W_{\Psi} f\right)(a, b)$ according to the space variable $b$ is:

$$
\begin{aligned}
\frac{\partial\left(W_{\Psi} f\right)(a, b)}{\partial b} & =\frac{1}{\sqrt{a}} \int_{-\infty}^{+\infty} f(t) \cdot \frac{\overline{\frac{\left(\Psi\left(\frac{t-b}{a}\right)\right.}{\partial b}} d t}{} \\
& =\frac{-1}{\sqrt{a^{3}}} \int_{-\infty}^{+\infty} f(t) \cdot \overline{\Psi^{\prime}\left(\frac{t-b}{a}\right)} d t
\end{aligned}
$$


As $\Psi$ is a compactly supported wavelet, using partial integration, the above quantity becomes:

$$
\frac{\partial\left(W_{\Psi} f\right)(a, b)}{\partial b}=\frac{1}{\sqrt{a}} \int_{-\infty}^{+\infty} f^{\prime}(t) \cdot \overline{\Psi\left(\frac{t-b}{a}\right)} d t
$$

The Equation 16.1 is equivalent to

$$
\frac{\partial\left|\left(W_{\Psi} f\right)(a, b)\right|^{2}}{\partial b}=2 \operatorname{Re}\left(\frac{\partial\left(W_{\Psi} f\right)(a, b)}{\partial b} \overline{\left(W_{\Psi} f\right)(a, b)}\right) ;
$$

the expression of

$$
\frac{\partial\left(W_{\Psi} f\right)(a, b)}{\partial b}
$$

in this equality leads to Equation 16.2 .

\section{Appendix 2: Approximation of CWT Square Modulus Derivative}

The quantity

$$
\int_{-\infty}^{+\infty} U(t) \cdot \overline{\Psi\left(\frac{t-b}{a}\right)} d t
$$

is equal to

$$
a \int_{-\infty}^{+\infty} U(a x+b) \cdot \overline{\Psi(x)} d x
$$

Note that the last integral holds only on the support of $\Psi$. Assuming that $U$ is differentiable, the above quantity can be approximated by:

$$
a \int_{-\infty}^{+\infty}\left(U(b)+a x U^{\prime}(b)\right) \cdot \overline{\Psi(x)} d x=a^{2} U^{\prime}(b) \int_{-\infty}^{+\infty} x \cdot \overline{\Psi(x)} d x
$$

( $\Psi$ is zero mean) for small values of $a$ (fine-scale analysis and then high frequencies). Assume now that $f^{\prime}$ is differentiable, one can then use this last approximation for each term in the right-hand part of Equation 16.2:

$$
\begin{aligned}
& \int_{-\infty}^{+\infty} f^{\prime}(t) \cdot \overline{\Psi\left(\frac{t-b}{a}\right)} d t \approx a^{2} f^{\prime \prime}(b) \int_{-\infty}^{+\infty} x \cdot \overline{\Psi(x)} d x \\
& \int_{-\infty}^{+\infty} f(t) \cdot \overline{\Psi\left(\frac{t-b}{a}\right)} d t \approx a^{2} f^{\prime}(b) \int_{-\infty}^{+\infty} x \cdot \overline{\Psi(x)} d x
\end{aligned}
$$


which permits obtaining Equation 16.3.

\section{Appendix 3: Expression of $\left(\boldsymbol{W}_{\boldsymbol{\Psi}} \boldsymbol{f}\right)\left(a, b_{0}\right)$}

1. Case of: $\left(\exists b_{0} \in \mathbb{R}\right)(\exists \varepsilon>0)(\forall|h|<\varepsilon) f\left(b_{0}+h\right)=f\left(b_{0}-h\right)$

Using a change variable we obtain:

$$
\begin{gathered}
\left(W_{\Psi} f\right)\left(a, b_{0}\right)=\sqrt{a} \cdot \int_{-\infty}^{+\infty} f\left(a t+b_{0}\right) \overline{\Psi(t)} d t \\
=\sqrt{a} \cdot \int_{0}^{+\infty}\left(f\left(a t+b_{0}\right)+f\left(-a t+b_{0}\right)\right) \cdot \operatorname{Re}(\Psi(t)) d t .
\end{gathered}
$$

As $\Psi$ is hermitian and compactly supported, its definition domain has the form $(-\Delta / 2, \Delta / 2)$. Hence, for all values $a$ such that $a \Delta / 2<$ $\varepsilon$ we have $f\left(a t+b_{0}\right)=f\left(-a t+b_{0}\right)$. Based on this remark and using a simple variable change one can obtain the Equation 16.5.

2. Case of: $\left(\exists b_{0} \in \mathbb{R}\right)(\exists \varepsilon>0)(\forall|h|<\varepsilon) f\left(b_{0}+h\right)=2 f\left(b_{0}\right)-f\left(b_{0}-h\right)$ The same approach is adopted: for $a \Delta / 2<\varepsilon$ we use the above relation and the zero mean property of the wavelet to obtain Equation 16.6.

\section{References}

[1] P. Trahanias and E. Skordalakis. Syntactic pattern recognition of the ECG. IEEE Trans. PAMI 12(7), 648-657, 1990.

[2] O. Meste, H. Rix, P. Caminal, and N. V. Thakor. Detection of late potentials by means of wavelet transform. IEEE Trans. BME 41(7), 625-634, 1994.

[3] R. Murray, S. Kadambe, and G. F. Boudreaux-Bartels. Extensive analysis of a QRS detector based on the dyadic wavelet transform. Proc. IEEE-Sig. Process. Int. Symp. on Time-Frequency Time-Scale Analysis, 1994, 540-543.

[4] L. Senhadji, J. J. Bellanger, G. Carrault, and J. L. Coatrieux. Wavelet analysis of ECG signals. Proc. IEEE-EMBS, 1990, 811-812. 
[5] L. Senhadji, J. J. Bellanger, G. Carrault, and G. F. Passariello. Comparing wavelet transforms for recognizing cardiac patterns. IEEEEMB Mag. Special Issue, Time-Frequency and Wavelet Analysis, 14(2), 167-173, 1995.

[6] A. Grossmann and J. Morlet. Decomposition of hardy functions into square integrable wavelets of constant shape. SIAM J. Math. Anal., 15(4), 723-736, 1984.

[7] A. Grossmann, R. K. Martinet, and J. Morlet. Reading and understanding continuous wavelet transforms. Proc. Int. Conf. Wavelets, Time-Frequency Methods and Phase Space, Marseille, France. J. M. Combes, et al. Eds., Inverse Problems and Theoretical Imaging, Springer-Verlag, 1989, 2-20.

[8] S. G. Mallat. A theory of multiresolution signal decomposition: the wavelet representation. IEEE Trans. PAMI, 11(7), 674-693, 1989.

[9] I. Daubechies. Orthonormal basis of compactly supported wavelets. Commun. Pure Appl. Math., 41, 909-996, 1988.

[10] S. G. Mallat and S. Zhong. Characterization of signals from multiscale edges. IEEE Trans. PAMI, 14(7), 710-732, 1992.

[11] L. Thoraval. Analyse statistique de signaux ECG par modèles de Markov cachés. Ph.D. dissertation, University of Rennes I, France, July 1995.

[12] L. R. Rabiner. A tutorial on hidden Markov models and selected applications in speech recognition. Proc. IEEE, 77(2), 257-285,1989.

[13] L. Thoraval, G. Carrault, and J. J. Bellanger. Heart signal recognition by hidden Markov models: the ECG case. Meth. Inf. Med., 33(1), 1014, 1994. 